\title{
A Statistical Comparison of Anatomical Features in Some Ornithogalum SP. SPECIES ${ }^{1}$
}

\author{
Comparação Estatística das Características Anatômicas em Espécies de Ornithogalum sp.
}

\author{
ÖZDEMİR, A. ${ }^{2}$, KOCAYİGİT, H. ${ }^{2}$, YETIŞEN, K. ${ }^{3}$, AKYOL, Y. ${ }^{3}$, and ÖZDEMİR, C. ${ }^{3}$
}

\begin{abstract}
In the present study, Ornithogalum narbonense, O. montanum, O. wiedemannii, $O$. sigmoideum and $O$. lanceolatum species were compared statistically with respect to anatomical characters. Some differences were found in root, stem and leaf anatomy of the species. These differences and similarities were indicated in this study. A big metaxylem was present in all root cross sections, except for O. lanceolatum, which has three metaxylema. 2-3 layered annular type collenchyma were present in all species. Aerenchyma is present in all mesophiles.
\end{abstract}

Keywords: statistic, Ornithogalum, anatomy.

RESUMO - No presente estudo, as espécies Ornithogalum narbonense, O. montanum, O. wiedemannii, O. sigmoideum e O. lanceolatum, foram comparadas estatisticamente com relação às suas características anatômicas. Foram observadas diferenças na anatomia da raiz, caule e folhas das espécies. Estas diferenças e semelhanças foram indicadas no presente estudo. Foi observado um grande metaxilema em todas as seções da raiz, com exceção de $\mathbf{O}$. lanceolatum, que possui três metaxilemas. Duas a três camadas de colênquima foram encontradas em todas as espécies. Todas as espécies mesófilas continham aerênquima.

Palavras-chave: estatística, Ornithogalum, anatomia.

\section{INTRODUCTION}

The genus Ornithogalum belonging to Hyacinthaceae family contains over 140 species widespread around the world. The genus is mostly spread in South Africa and around the Mediterranean (Uysal et al., 2005). Ornithogalum is represented by 45 species in the flora of Turkey and 17 of these species are endemic (Cullen, 1984; Davis et al., 1988; Ozhatay, 2000; Uysal et al., 2005; Varol, 2008; Dalg1c et al., 2009). Ornithogalum is a taxonomically difficult genus, while its morphology is poorly correlated with the variation in chromosome number and karyotype (Dalg1c and Özhatay, 1997). Since several decades, this taxonomically extremely difficult genus was the object of several studies on bulb structure and germination-type (Zahariadi, 1962, 1965; Speta, 1990, 1990a), classical cytotaxonomy (Peruzzi and Passalacqua, 2002; Garbari et al., 2003; Tornadore et al., 2003; Aquaro and Peruzzi, 2006), karyotype evolution (van Raamsdonk, 1986), chemotaxonomy (Øvstedal, 1991), morphometry (Moret et al., 1991; Øvstedal, 1991; Raamsdonk and Heringa, 1987; Moret, 1992; Moret and Galland, 1992; Coskuncelebi et al., 2002), seed micromorphology (Coskuncelebi, et al., 2000). In this study, Ornithogalum narbonense, O. montanum, O. wiedemannii, $O$. sigmoideum and $O$. lanceolatum species were compared statistically regarding their anatomical characters.

Recebido para publicação em 1.9.2015 e aprovado em 5.2.2016.

2 Celal Bayar University, Faculty of Art and Science, Department of Mathematics; ${ }^{3}$ Celal Bayar University, Faculty of Art and Science, Department of Biology, 45030-Muradiye, Manisa,Turkey. <kadriyeyetisen@gmail.com>. 


\section{MATERIAL AND METHODS}

Materials were collected from B1 Manisa Spil Mountain. Specimens were kept in the herbarium at Celal Bayar University. A morphological illustration of the plant taxon was made from fresh and dry specimens follewed "Flora of Turkey" volume 8 (Davis, 1984). For anatomical studies, the plant specimens were fixed in $70 \%$ ethanol. Hand cuts were stained with sartur reactive and safranin. Preparates were photographed with a motorized Leica DM 3000 microscope. Measurements were taken using ocularmicrometer of root, stem and leaf cell sizes of the species. Minimum, maximum, mean and standart deviations were determined.

For the numerical analysis, 16 anatomical characters were selected. This selection was based on the variations of the anatomical data. Characters were coded as $1,2,3,4,5,6,7,8$, $9,10,11,12,13,14,15,16$ and the taxa were coded as A, B, C, D, E. Significance of the differences between the taxa and characters were evaluated by Analysis of variance (Regression Analysis) and Pearson's correlation. The differences were assessed by the one-way ANOVA test. Pearson's correlation coefficients were also calculated. Statistical analysis were performed using the MINITAB software package.

\section{RESULTS AND DISCUSSION}

\section{Anatomical findings}

\section{Roots}

$O$. narbonense and $O$. lanceolatum species have two layered epidermis. Other species have one layered epidermis. Cortex is 3-5, 3-4, 7-11, 6-8, 4-5 layered in O. narbonense, $O$. montanum, $O$. wiedemannii, $O$. sigmoedeum and $O$. lanceolatum, respectively. Four-sided endodermis wall thickening was present in O. narbonense, O. montanum and $O$. sigmoedeum. A big metaxylem was present in all root cross sections except for O. lanceolatum, which has three metaxylema. 4-5 xylem strands were present in all roots.

\section{Stems}

A thick cuticle surrounds the one layered epidermis in all stem cross sections. The cortex consists of circle shaped cells in all species and 4-5, 5-6, 2-3, 5-6, 4-5 layered in $O$. narbonense, $O$. montanum, $O$. wiedemannii, O. sigmoedeum, and O. lanceolatum, respectively. 2-3 layered annular type collenchyma were present in all species. Vascular bundles are arranged in two rows in $O$. narbonense and $O$. montanum, three rings in O. wiedemannii, O. sigmoedeum, and O. lanceolatum. Raphide crystals were present in the stem cortex of $O$. sigmoedeum.

\section{Leaves}

In all species, the cuticle is present on both sides of the leaf. O. narbonense, $O$. wiedemannii and $O$. lanceolatum leaves have 1-2 layered palisade parenchyma under adaxial and abaxial epidermis and 2-3 layered spongy parenchyma. Palisade spongy separation was absent in O. montanum and O. sigmoedeum species. Mesophiles of all species have aerenchyma. O. lanceolatum mesophill have bigger aerenchyma than others.

\section{Statistical analysis}

The anatomical measuments of the investigated taxa are shown in Table 1. Significance of the differences between the Ornithogalum taxa was evaluated by analysis of variance (Regression Analysis) and Pearson's correlation (Correlation). The statistical analysis of the results are shown in Tables 2, 3, 4, 5 and 6.

The differences among the investigated taxa are shown as A-C, A-D, A-E, B-C, B-D, D$\mathrm{E}$ and $\mathrm{C}-\mathrm{D}$ in Table 3 and Table 5, and they are significant at level of $0.01 \mathrm{P}$ and 0.05P. According to Table 4 and Table 6 , based on the Pearson's correlation method (Correlation), there are important correlations among the anatomical characters of the investigatigated taxa, shown as (Table 2) 1-3, 1-5, 1-7, 1-16, 215, 3- 4, 3-7, 3-12, 3-14, 3-16, 4-16, 6-10, 7-12, 8-12, 8-14, 9-15, 11-13 and $14-15$ at levels of $0.01 \mathrm{P}$ and $0.05 \mathrm{P}$.

The results of the statistical analysis are shown in Tables 4 and 6 . It was found that there were statistically important differences at levels of $0.01 \mathrm{P}$ and $0.05 \mathrm{P}$. 
Table 1 - Anatomical measurements of the species

\begin{tabular}{|c|c|c|c|c|c|}
\hline O. narbonense & Min.-Max & Ort \pm S.D & O. lanceolatum & Min.-Max. & Ort \pm S.D \\
\hline Root & & & Root & & \\
\hline Epidermis Width & $20-50$ & $35 \pm 13$ & Epidermis Width & $38-52$ & $47.5 \pm 6.4$ \\
\hline Epidermis Length & $15-50$ & $30 \pm 12$ & Epidermis Length & $30-50$ & $42 \pm 8$ \\
\hline Cortex (diameter) & $70-23$ & $140 \pm 62$ & Cortex (diameter) & $30-170$ & $100 \pm 99$ \\
\hline Endodermis Width & $18-23$ & $21 \pm 1.8$ & Endodermis Width & $12-22$ & $17.8 \pm 3.7$ \\
\hline Endodermis Length & $10-15$ & $12 \pm 1.8$ & Endodermis Length & $7-12$ & $10 \pm 1.7$ \\
\hline Pericycle Width & $15-18$ & $17 \pm 1.2$ & Pericycle Width & $15-26$ & $21 \pm 4.6$ \\
\hline Metaxylem ( diameter ) & $25-63$ & $38 \pm 13$ & Metaxylem (diameter) & $15-26$ & $21 \pm 4.6$ \\
\hline Stem & & & Stem & & \\
\hline Epidermis Width & $13-25$ & $17 \pm 2.5$ & Epidermis Width & $18-22$ & $20 \pm 1.6$ \\
\hline Epidermis Length & $30-43$ & $35 \pm 4.3$ & Epidermis Length & $18-23$ & $20.6 \pm 2$ \\
\hline Cortex (diameter) & $3-50$ & $48 \pm 9.7$ & Cortex (diameter) & $20-70$ & $45 \pm 14.5$ \\
\hline Trachea (diameter) & $8-23$ & $19 \pm 2.5$ & Trachea (diameter) & $10-15$ & $12 \pm 2.7$ \\
\hline Leaf & & & Leaf & & \\
\hline Abaxial epidermis Width & $38-50$ & $44 \pm 5.6$ & Abaxial epidermis Width & $10-22$ & $20 \pm 0.8$ \\
\hline Abaxial epidermis Length & $20-28$ & $26 \pm 3.3$ & Abaxial epidermis Length & $25-30$ & $29 \pm 2.2$ \\
\hline Palisade Length & $28-40$ & $34 \pm 4.7$ & Palisade Length & $20-25$ & $22.8 \pm 2.16$ \\
\hline Adaxial epidermis Width & $18-25$ & $22 \pm 2.5$ & Adaxial epidermis Width & $12-25$ & $18.8 \pm 4.5$ \\
\hline Adaxial epidermis Length & $30-38$ & $34 \pm 2.5$ & Adaxial epidermis Length & $12-20$ & $16 \pm 4$ \\
\hline O. sigmoideum & Min.-Max & Ort \pm S.D & O. wiedemannii var. wiedemannii & Min.-Max. & Ort \pm S.D \\
\hline Root & & & Root & & \\
\hline Epidermis Width & $15-38$ & $24.5 \pm 9.8$ & Epidermis Width & $10-20$ & $15 \pm 5$ \\
\hline Epidermis Length & $20-50$ & $32 \pm 13.8$ & Epidermis Length & $20-40$ & $30 \pm 10$ \\
\hline Cortex (diameter) & $10-35$ & $25 \pm 10$ & Cortex (diameter) & $22-10$ & $16 \pm 6$ \\
\hline Endodermis Width & $18-23$ & $20.6 \pm 2$ & Endodermis Width & $5-15$ & $9.6 \pm 5$ \\
\hline Endodermis Length & $11-18$ & $14.6 \pm 3.5$ & Endodermis Length & $10-18$ & $13 \pm 3$ \\
\hline Pericycle Width & $10-18$ & $15 \pm 3.4$ & Pericycle Width & $15-25$ & $20 \pm 5$ \\
\hline Metaxylem (diameter) & $35-40$ & $38 \pm 2.1$ & Metaxylem (diameter) & & \\
\hline Stem & & & Stem & $30-38$ & $34 \pm 3$ \\
\hline Epidermis Width & $20-35$ & $26 \pm 6.5$ & Epidermis Width & $25-30$ & $26.5 \pm 2$ \\
\hline Epidermis Length & $30-40$ & $37 \pm 4.4$ & Epidermis Length & $28-88$ & $72 \pm 22$ \\
\hline Cortex (diameter) & $35-110$ & $64 \pm 31$ & Cortex (diameter) & $25-38$ & $33.5 \pm 5$ \\
\hline Trachea (diameter) & $15-30$ & $23 \pm 6.7$ & Trachea (diameter) & & \\
\hline Leaf & & & Leaf & $15-23$ & $20 \pm 5.1$ \\
\hline Abaxial epidermis Width & $25-40$ & $32.5 \pm 6.4$ & Abaxial epidermis Width & $15-20$ & $17.5 \pm 2$ \\
\hline Abaxial epidermis Length & $20-25$ & $23 \pm 2.4$ & Abaxial epidermis Length & $63-70$ & $66 \pm 2.8$ \\
\hline Palisade Length & $40-170$ & $83.5 \pm 59$ & Palisade Length & $12-25$ & $19 \pm 4.8$ \\
\hline Adaxial epidermis Width & $13-20$ & $16.3 \pm 3$ & Adaxial epidermis Width & $15-22.5$ & $17 \pm 3.2$ \\
\hline Adaxial epidermis Length & $20-27$ & $23 \pm 2.6$ & Adaxial epidermis Length & $10-20$ & $15 \pm 5$ \\
\hline O. montanum & Min.-Max & Ort \pm S.D & & & \\
\hline Root & $20-45$ & $37 \pm 9.7$ & & & \\
\hline Epidermis Width & $10-30$ & $24 \pm 9$ & & & \\
\hline Epidermis Length & $40-60$ & $53 \pm 8.3$ & & & \\
\hline Cortex (diameter) & $13-15$ & $14 \pm 0.9$ & & & \\
\hline Endodermis Width & $7-10$ & $8.5 \pm 1.2$ & & & \\
\hline Endodermis Length & $9-11$ & $10 \pm 0.8$ & & & \\
\hline Pericycle Width & $20-32$ & $27 \pm 0.4$ & & & \\
\hline \multicolumn{3}{|l|}{ Metaxylem (diameter) } & & & \\
\hline Stem & $15-20$ & $17 \pm 2.5$ & & & \\
\hline Epidermis Width & $10-25$ & $19 \pm 6.2$ & & & \\
\hline Epidermis Length & $15-40$ & $33.6 \pm 16$ & & & \\
\hline Cortex (diameter) & $3-15$ & $7.6 \pm 4.8$ & & & \\
\hline \multicolumn{3}{|l|}{ Trachea (diameter) } & & & \\
\hline Leaf & $10-28$ & $20 \pm 7.5$ & & & \\
\hline Abaxial epidermis Width & $20-40$ & $27.5 \pm 8.6$ & & & \\
\hline Abaxial epidermis Length & $38-15$ & $33 \pm 3$ & & & \\
\hline Palisade Length & $15-28$ & $22 \pm 5$ & & & \\
\hline Adaxial epidermis Width & $30-42$ & $38 \pm 4.5$ & & & \\
\hline Adaxial epidermis Length & $20-45$ & $37 \pm 9.7$ & & & \\
\hline
\end{tabular}


Table 2 - Pearson's correlation (Correlation) based on anatomical characters of the investigated taxa

\begin{tabular}{|c|c|c|c|c|c|c|c|c|c|c|c|c|c|c|c|}
\hline Features & 1 & 2 & 3 & 4 & 5 & 6 & 7 & 8 & 9 & 10 & 11 & 12 & 13 & 14 & 15 \\
\hline \multirow{2}{*}{1} & 0.607 & & & & & & & & & & & & & & \\
\hline & 0.278 & & & & & & & & & & & & & & \\
\hline \multirow{2}{*}{2} & 0.962 & 0.744 & & & & & & & & & & & & & \\
\hline & $0.009 * *$ & 0.150 & & & & & & & & & & & & & \\
\hline \multirow{2}{*}{3} & 0.948 & 0.691 & 0.947 & & & & & & & & & & & & \\
\hline & 0.014 & 0.196 & $0.015^{*}$ & & & & & & & & & & & & \\
\hline \multirow{2}{*}{4} & $0.041 *$ & 0.798 & 0.204 & 0.220 & & & & & & & & & & & \\
\hline & 0.948 & 0.106 & 0.742 & 0.722 & & & & & & & & & & & \\
\hline \multirow{2}{*}{5} & 0.343 & 0.244 & -0.138 & 0.055 & 0.565 & & & & & & & & & & \\
\hline & 0.572 & 0.692 & 0.825 & 0.930 & 0.320 & & & & & & & & & & \\
\hline \multirow{2}{*}{6} & 0.962 & 0.396 & 0.894 & 0.851 & 0.222 & 0.492 & & & & & & & & & \\
\hline & $0.009 * *$ & 0.510 & $0.041 *$ & 0.068 & 0.720 & 0.400 & & & & & & & & & \\
\hline \multirow{2}{*}{7} & 0.874 & 0.721 & 0.872 & 0.745 & 0.221 & 0.456 & 0.819 & & & & & & & & \\
\hline & 0.053 & 0.169 & 0.054 & 0.149 & 0.721 & 0.441 & 0.090 & & & & & & & & \\
\hline \multirow{2}{*}{8} & 0.649 & 0.858 & 0.693 & 0.808 & 0.703 & 0.249 & 0.421 & 0.564 & & & & & & & \\
\hline & 0.236 & 0.063 & 0.194 & 0.098 & 0.186 & 0.686 & 0.480 & 0.322 & & & & & & & \\
\hline \multirow{2}{*}{9} & 0.751 & 0.415 & 0.667 & 0.869 & 0.118 & 0.011* & 0.649 & 0.414 & 0.773 & & & & & & \\
\hline & 0.144 & 0.487 & 0.219 & 0.056 & 0.850 & 0.985 & 0.236 & 0.488 & 0.126 & & & & & & \\
\hline \multirow{2}{*}{10} & 0.873 & 0.622 & 0.778 & 0.857 & 0.241 & 0.371 & 0.762 & 0.795 & 0.797 & 0.820 & & & & & \\
\hline & 0.053 & 0.262 & 0.121 & 0.064 & 0.696 & 0.539 & 0.134 & 0.108 & 0.107 & 0.089 & & & & & \\
\hline \multirow{2}{*}{11} & 0.975 & 0.542 & 0.917 & 0.856 & 0.045 & 0.517 & 0.970 & 0.927 & 0.525 & 0.620 & 0.847 & & & & \\
\hline & 0.005 & 0.345 & $0.028 *$ & 0.064 & 0.942 & 0.372 & $0.006 * *$ & $0.023 *$ & 0.363 & 0.264 & 0.070 & & & & \\
\hline \multirow{2}{*}{12} & 0.791 & 0.173 & 0.610 & 0.755 & 0.237 & 0.496 & 0.791 & 0.527 & 0.500 & 0.870 & 0.854 & 0.760 & & & \\
\hline & 0.111 & 0.781 & 0.274 & 0.140 & 0.701 & 0.395 & 0.111 & 0.362 & 0.391 & 0.055 & 0.066 & 0.136 & & & \\
\hline \multirow{2}{*}{13} & \begin{tabular}{|l}
0.910 \\
\end{tabular} & 0.730 & 0.928 & 0.800 & 0.194 & 0.382 & 0.863 & 0.988 & 0.570 & 0.451 & 0.770 & 0.944 & 0.528 & & \\
\hline & 0.032 & 0.162 & $0.023 *$ & 0.104 & 0.755 & 0.526 & 0.060 & $0.002 * *$ & 0.316 & 0.446 & 0.128 & $0.016^{*}$ & 0.361 & & \\
\hline \multirow{2}{*}{14} & 0.737 & 0.959 & 0.804 & 0.809 & 0.700 & 0.082 & 0.533 & 0.785 & 0.937 & 0.614 & 0.814 & 0.672 & 0.429 & 0.781 & \\
\hline & 0.155 & $0.010^{*}$ & 0.101 & 0.097 & 0.188 & 0.896 & 0.355 & 0.116 & 0.019* & 0.270 & 0.094 & 0.214 & 0.471 & 0.119 & \\
\hline \multirow{2}{*}{15} & 0.932 & 0.848 & 0.976 & 0.953 & 0.385 & 0.066 & 0.811 & 0.873 & 0.825 & 0.707 & 0.843 & 0.874 & 0.600 & 0.910 & 0.913 \\
\hline & $0.021 *$ & 0.070 & $0.005^{* * *}$ & $0.012 *$ & 0.522 & 0.915 & 0.096 & 0.053 & 0.085 & 0.182 & 0.073 & 0.053 & 0.285 & $0.032 *$ & $0.030^{*}$ \\
\hline
\end{tabular}

* Significant at the level of 0.05 . ** Significant at the level of 0.01 .

Table 3 - Pearson correlation (Correlation) based on 8 anatomical characters of the leaves of the investigated taxa

\begin{tabular}{|c|l|l|l|l|}
\hline Characters & \multicolumn{1}{|c|}{$\mathrm{A}$} & $\mathrm{B}$ & $\mathrm{C}$ & $\mathrm{D}$ \\
\hline \multirow{2}{*}{$\mathrm{B}$} & 0.362 & & & \\
\cline { 2 - 5 } & 0.140 & & & \\
\hline \multirow{2}{*}{$\mathrm{C}$} & $0.006^{* *}$ & 0.573 & & \\
\hline & 0.979 & $0.013^{*}$ & & \\
\hline \multirow{2}{*}{$\mathrm{D}$} & $0.007^{* *}$ & 0.483 & 0.749 & \\
\cline { 2 - 5 } & 0.978 & $0.042 *$ & $0.008^{* *}$ & \\
\hline \multirow{2}{*}{$\mathrm{E}$} & $0.029^{*}$ & 0.194 & 0.430 & $0.030^{*}$ \\
\cline { 2 - 5 } & 0.910 & 0.441 & 0.075 & 0.906 \\
\hline
\end{tabular}

$O$. narbonense (A), O. lanceolatum (B), O. sigmoideum (C), O. wiedemannii (D), O. montanum (E), O. wiedemannii (D), O. montanum (E)
In the present study, five Ornithogalum taxa were compared statistically regarding anatomical characters. We aimed to find statistical and anatomical differences in addition to morphological characters to distinguish these species. Anatomical features of the root of the five species are similar. O. lanceolatum have three metaxylema in the root while others have one metaxylem. Scape has a single layered epidermis with a thin cuticle in all taxa. In most species of the subfamily Ornithogaloideae, vascular bundles are in two rows and large bundles alternate with small bundles (Lynch et al., 2006). In our study, vascular bundles are in two rows in 
Table 4 - Comparison anatomical features of the investigated taxa with Minitab

\begin{tabular}{|c|c|c|c|c|c|c|c|c|}
\hline Takson & 1 & 2 & 3 & 4 & 5 & 6 & 7 & 8 \\
\hline \multirow{2}{*}{1} & 0.582 & & & & & & & \\
\hline & 0.303 & & & & & & & \\
\hline \multirow{2}{*}{2} & 0.546 & 0.998 & & & & & & \\
\hline & 0.341 & $0.010 * *$ & & & & & & \\
\hline \multirow{2}{*}{3} & 0.957 & 0.768 & 0.736 & & & & & \\
\hline & $0.010 * *$ & 0.130 & 0.156 & & & & & \\
\hline \multirow{2}{*}{4} & 0.873 & 0.516 & 0.472 & 0.841 & & & & \\
\hline & 0.053 & 0.373 & 0.422 & 0.074 & & & & \\
\hline \multirow{2}{*}{5} & 0.759 & 0.932 & 0.925 & 0.852 & 0.688 & & & \\
\hline & 0.136 & $0.021 *$ & $0.024^{*}$ & 0.067 & 0.199 & & & \\
\hline \multirow{2}{*}{6} & -0.057 & 0.458 & 0.447 & 0.202 & 0.028 & 0.136 & & \\
\hline & 0.927 & 0.438 & 0.450 & 0.744 & 0.965 & 0.827 & & \\
\hline \multirow{2}{*}{7} & 0.996 & 0.613 & 0.576 & 0.967 & 0.909 & 0.780 & -0.007 & \\
\hline & $0.020 *$ & 0.272 & 0.310 & $0.007 * *$ & 0.032 & 0.120 & 0.991 & \\
\hline \multirow{2}{*}{8} & 0.865 & 0.547 & 0.537 & 0.787 & 0.619 & 0.778 & -0.382 & 0.833 \\
\hline & 0.058 & 0.340 & 0.351 & 0.114 & 0.265 & 0.121 & 0.526 & 0.080 \\
\hline
\end{tabular}

Table 5 - Comparison anatomical features of the investigated taxa with Pearson's correlation analysis

\begin{tabular}{|c|l|l|l|l|}
\hline Taxa & $\mathrm{C} 1$ & $\mathrm{C} 2$ & $\mathrm{C} 3$ & $\mathrm{C} 4$ \\
\hline \multirow{2}{*}{$\mathrm{C} 2$} & 0.631 & & & \\
\cline { 2 - 5 } & 0.009 & & & \\
\hline \multirow{2}{*}{$\mathrm{C} 3$} & 0.211 & 0.503 & & \\
\cline { 2 - 5 } & 0.433 & $0.047^{*}$ & & \\
\hline \multirow{2}{*}{$\mathrm{C} 4$} & 0.125 & 0.192 & 0.177 & \\
\cline { 2 - 5 } & 0.644 & 0.476 & 0.511 & \\
\hline \multirow{2}{*}{$\mathrm{C} 5$} & 0.621 & 0.730 & 0.143 & 0.103 \\
\cline { 2 - 5 } & $0.010^{*}$ & $0.001^{* *}$ & 0.597 & 0.705 \\
\hline
\end{tabular}

O. narbonense and $O$. montanum are in three rows in O. wiedemannii var wiedemannii, O.sigmoedeum, and O. lanceolatum. Lynch et al. (2006) reported that Hyacinthaceae has a wide range of variation in the crystal types. There are raphide, styloid and druse crystals in this family (Lynch et al., 2006). Raphide crystals are present in the stem cortex of O.sigmoedeum. The presence, morphology and distribution of crystals within a species are very important, because it is under genetic control (Franceschi and Nakata, 2005). Thus, the constancy of crystal type and distribution can be viewed as a taxonomic character for classification of species. Leaf anatomy of the taxa shows some
Table 6 - Comparison anatomical features of the investigated taxa with Minitab

\begin{tabular}{|c|c|c|c|c|c|}
\hline \multicolumn{6}{|c|}{ One - way ANOVA: $\mathrm{C} 1$ versus $\mathrm{C} 2$} \\
\hline Source & DF & SS & MS & $\mathbf{F}$ & $\mathbf{P}$ \\
\hline $\mathrm{C} 2$ & 12 & 9651 & 804 & 0.52 & 0.817 \\
\hline Error & 3 & 4597 & 1532 & & \\
\hline Total & 15 & 14247 & & & \\
\hline \multicolumn{3}{|c|}{$S=39.14$} & \multicolumn{3}{|c|}{$\mathrm{R}-\mathrm{Sq}(\operatorname{adj})=0.00 \%$} \\
\hline Level & $\mathbf{N}$ & & Mean & & StDev \\
\hline 12 & 1 & & 19.00 & & $\wedge$ \\
\hline 13 & 1 & & 17.00 & & $\wedge$ \\
\hline 16 & 1 & & 34.00 & & $\wedge$ \\
\hline 17 & 1 & & 21.00 & & $\wedge$ \\
\hline 18 & 1 & & 25.00 & & $\wedge$ \\
\hline 20 & 2 & & 30.00 & & 19.09 \\
\hline 21 & 1 & & 38.00 & & $\wedge$ \\
\hline 22 & 1 & & 34.00 & & $\wedge$ \\
\hline 25 & 1 & & 40.00 & & $\wedge$ \\
\hline 45 & 2 & & 94.00 & & 65.05 \\
\hline 47 & 2 & & 35.00 & & 0.00 \\
\hline 51 & 1 & & 70.00 & & $\wedge$ \\
\hline 55 & 1 & & 75.00 & & $\wedge$ \\
\hline
\end{tabular}

Pooled StDev $=39.14$.

To be continued... 
Table 6, cont...

\begin{tabular}{|l|c|c|c|c|c|}
\hline \multicolumn{5}{|c|}{ One - way ANOVA: C1 versus C3 } \\
\hline Source & DF & SS & MS & F & P \\
\hline C3 & 12 & 8920 & 743 & 0.42 & 0.880 \\
\hline Error & 3 & 5327 & 1762 & & \\
\hline Total & 15 & 14247 & & & \\
\hline \multicolumn{2}{|c|}{ S=42.14 } & R-Sq=62.61 \% & R-Sq(adj) $=0.00 \%$ \\
\hline Level & N & \multicolumn{3}{|c|}{ Mean } & StDev \\
\hline 15 & 1 & \multicolumn{3}{|c|}{17.00} & $\wedge$ \\
\hline 16 & 1 & 25.00 & $\wedge$ \\
\hline 20 & 1 & 21.00 & $\wedge$ \\
\hline 23 & 1 & 26.00 & 10.61 \\
\hline 24 & 1 & 35.00 & $\wedge$ \\
\hline 25 & 2 & 89.00 & 72.12 \\
\hline 26 & 1 & \multicolumn{2}{|c|}{17.00} & $\wedge$ \\
\hline 32 & 1 & 44.00 & $\wedge$ \\
\hline 50 & 2 & 37.00 & 3.54 \\
\hline 55 & 1 & 75.00 & $\wedge$ \\
\hline 64 & 1 & 48.00 & $\wedge$ \\
\hline 68 & 1 & 70.00 & $\wedge$ \\
\hline 82 & 1 & 34.00 & $\wedge$ \\
\hline
\end{tabular}

Pooled StDev $=42.14$.

\begin{tabular}{|l|c|c|c|c|c|}
\hline \multicolumn{6}{|c|}{ One - way ANOVA: C1 versus C5 } \\
\hline Source & DF & SS & MS & F & P \\
\hline C5 & 12 & 8732 & 728 & 0.40 & 0.893 \\
\hline Error & 3 & 5515 & 1838 & & \\
\hline Total & 15 & 14247 & & & \\
\hline \multicolumn{2}{|c|}{ S=42.88 } & R-Sq=62.29 \% & R-Sq(adj) $=0.00 \%$ \\
\hline Level & N & \multicolumn{3}{|c|}{ Mean } & StDev \\
\hline 7 & 1 & \multicolumn{2}{|c|}{17.00} & $\wedge$ \\
\hline 10 & 1 & 25.00 & $\wedge$ \\
\hline 11 & 1 & 21.00 & $\wedge$ \\
\hline 14 & 1 & 26.00 & $\wedge$ \\
\hline 15 & 2 & 35.00 & 1.41 \\
\hline 17 & 1 & 89.00 & 72.12 \\
\hline 20 & 1 & 17.00 & $\wedge$ \\
\hline 22 & 1 & 44.00 & $\wedge$ \\
\hline 25 & 2 & 37.00 & $\wedge$ \\
\hline 33 & 1 & 75.00 & $\wedge$ \\
\hline 37 & 2 & 48.00 & 0.71 \\
\hline 40 & 1 & 70.00 & $\wedge$ \\
\hline 53 & 2 & 34.00 & $\wedge$ \\
\hline
\end{tabular}

Pooled StDev $=42.88$.

To be continued...
Table 6, cont...

\begin{tabular}{|l|c|c|c|c|c|}
\hline \multicolumn{5}{|c|}{ One - way ANOVA: C2 versus C3 } \\
\hline Source & DF & SS & MS & F & P \\
\hline C3 & 12 & 3046 & 254 & 1.42 & 0.434 \\
\hline Error & 3 & 538 & 179 & & \\
\hline Total & 15 & 3584 & & & \\
\hline S=13.39 & R-Sq=84.99 \% & \multicolumn{2}{c|}{ R-Sq(adj) $=24.94 \%$} \\
\hline Level & N & \multicolumn{2}{|c|}{ Mean } & StDev \\
\hline 15 & 1 & \multicolumn{2}{|c|}{13.00} & $\wedge$ \\
\hline 16 & 1 & 18.00 & $\wedge$ \\
\hline 20 & 1 & 17.00 & $\wedge$ \\
\hline 22 & 2 & 14.00 & 2.82 \\
\hline 24 & 1 & 47.00 & $\wedge$ \\
\hline 25 & 1 & 33.00 & 16.97 \\
\hline 26 & 1 & 20.00 & $\wedge$ \\
\hline 32 & 1 & 20.00 & $\wedge$ \\
\hline 50 & 2 & 36.00 & 15.56 \\
\hline 55 & 1 & 55.00 & $\wedge$ \\
\hline 64 & 1 & 45.00 & $\wedge$ \\
\hline 68 & 1 & 51.00 & $\wedge$ \\
\hline 82 & 1 & 22.00 & $\wedge$ \\
\hline
\end{tabular}

Pooled StDev $=13.39$.

\begin{tabular}{|l|c|r|r|r|c|}
\hline \multicolumn{6}{|c|}{ One - way ANOVA: C2 versus C4 } \\
\hline Source & DF & \multicolumn{1}{|c|}{ SS } & MS & F & P \\
\hline C4 & 12 & 3542 & 295 & 21.6 & 0.014 \\
\hline Error & 3 & 41 & 13 & & \\
\hline Total & 15 & 3582 & & & \\
\hline \multicolumn{2}{|c|}{ S=3.697 } & R-Sq=98.86 \% & \multicolumn{2}{c|}{ R-Sq(adj) $=94.28 \%$} \\
\hline Level & N & \multicolumn{2}{|c|}{ Mean } & StDev \\
\hline 12 & 1 & \multicolumn{2}{|c|}{12.00} & $\wedge$ \\
\hline 15 & 2 & 47.00 & 0.000 \\
\hline 16 & 1 & 17.00 & $\wedge$ \\
\hline 19 & 2 & 22.00 & $\wedge$ \\
\hline 20 & 2 & 20.00 & 0.707 \\
\hline 25 & 2 & 20.00 & 6.364 \\
\hline 30 & 1 & 45.00 & $\wedge$ \\
\hline 33 & 1 & 12.00 & $\wedge$ \\
\hline 34 & 1 & 20.00 & $\wedge$ \\
\hline 35 & 1 & 55.00 & $\wedge$ \\
\hline 40 & 1 & 51.00 & $\wedge$ \\
\hline 66 & 1 & 18.00 & $\wedge$ \\
\hline 72 & 1 & 45.00 & $\wedge$ \\
\hline
\end{tabular}


Table 6, cont...

\begin{tabular}{|c|c|c|c|c|c|}
\hline \multicolumn{5}{|c|}{ One - way ANOVA: C2 versus C5 } \\
\hline Source & DF & SS & MS & F & P \\
\hline C5 & 12 & 3092 & 258 & 1.58 & 0.392 \\
\hline Error & 3 & 491 & 164 & & \\
\hline Total & 15 & 3584 & & & \\
\hline S=12.79 & R-Sq=56.21 \% & \multicolumn{2}{c|}{ R-Sq(adj) $=31.57 \%$} \\
\hline Level & N & \multicolumn{2}{|c|}{ Mean } & StDev \\
\hline 7 & 1 & \multicolumn{3}{|c|}{12.00} & $\wedge$ \\
\hline 10 & 1 & 13.00 & $\wedge$ \\
\hline 11 & 1 & 22.00 & $\wedge$ \\
\hline 14 & 1 & 17.00 & $\wedge$ \\
\hline 15 & 2 & 23.00 & 2.83 \\
\hline 17 & 1 & 20.00 & $\wedge$ \\
\hline 20 & 1 & 20.00 & $\wedge$ \\
\hline 22 & 1 & 18.00 & $\wedge$ \\
\hline 25 & 1 & 55.00 & $\wedge$ \\
\hline 33 & 1 & 45.00 & $\wedge$ \\
\hline 37 & 2 & 31.00 & 21.92 \\
\hline 40 & 1 & 51.00 & $\wedge$ \\
\hline 53 & 2 & 46.00 & $\wedge$ \\
\hline
\end{tabular}

Pooled StDev $=12.79$.

\begin{tabular}{|l|c|c|c|c|c|}
\hline \multicolumn{5}{|c|}{ One - way ANOVA: C3 versus C4 } \\
\hline Source & DF & SS & MS & F & P \\
\hline C4 & 12 & 5947 & 496 & 2.05 & 0.304 \\
\hline Error & 3 & 727 & 242 & & \\
\hline Total & 15 & 6674 & & & \\
\hline S=12.79 & R-Sq=56.21 \% & \multicolumn{2}{|c|}{ R-Sq(adj) $=31.57 \%$} \\
\hline Level & N & \multicolumn{3}{|c|}{ Mean } & StDev \\
\hline 12 & 1 & \multicolumn{3}{|c|}{15.00} & $\wedge$ \\
\hline 15 & 2 & 37.00 & 18.38 \\
\hline 16 & 1 & 20.00 & $\wedge$ \\
\hline 19 & 1 & 83.00 & $\wedge$ \\
\hline 20 & 2 & 28.50 & 4.95 \\
\hline 25 & 2 & 36.50 & 19.09 \\
\hline 30 & 1 & 25.00 & $\wedge$ \\
\hline 33 & 1 & 23.00 & $\wedge$ \\
\hline 34 & 1 & 26.00 & $\wedge$ \\
\hline 35 & 1 & 55.00 & $\wedge$ \\
\hline 40 & 1 & 68.00 & $\wedge$ \\
\hline 66 & 1 & 16.00 & $\wedge$ \\
\hline 72 & 1 & 64.00 & $\wedge$ \\
\hline
\end{tabular}

Pooled StDev $=15.57$.

To be continued...
Table 6, cont...

\begin{tabular}{|l|c|c|c|c|c|}
\hline \multicolumn{5}{|c|}{ One - way ANOVA: C3 versus C5 } \\
\hline Source & DF & SS & MS & F & P \\
\hline C5 & 12 & 6048 & 504 & 2.42 & 0.254 \\
\hline Error & 3 & 626 & 209 & & \\
\hline Total & 15 & 6674 & & & \\
\hline S=14.44 & R-Sq=90.63 \% & R-Sq(adj) $=53.14 \%$ \\
\hline Level & N & \multicolumn{3}{|c|}{ Mean } & StDev \\
\hline 7 & 1 & \multicolumn{3}{|c|}{23.00} & $\wedge$ \\
\hline 10 & 1 & 15.00 & $\wedge$ \\
\hline 11 & 1 & 83.00 & $\wedge$ \\
\hline 14 & 1 & 20.00 & $\wedge$ \\
\hline 15 & 2 & 37.50 & 17.68 \\
\hline 17 & 1 & 26.00 & $\wedge$ \\
\hline 20 & 1 & \multicolumn{2}{|c|}{32.00} & $\wedge$ \\
\hline 22 & 1 & 16.00 & $\wedge$ \\
\hline 25 & 1 & 55.00 & $\wedge$ \\
\hline 33 & 1 & 64.00 & $\wedge$ \\
\hline 37 & 2 & 23.00 & $\wedge$ \\
\hline 40 & 1 & 68.00 & $\wedge$ \\
\hline 53 & 1 & 37.00 & $\wedge$ \\
\hline
\end{tabular}

Pooled StDev $=14.44$.

\begin{tabular}{|l|c|c|c|c|c|}
\hline \multicolumn{6}{|c|}{ One - way ANOVA: C4 versus C5 } \\
\hline Source & DF & SS & MS & F & P \\
\hline C5 & 12 & 4340 & 361 & 6.20 & 0.080 \\
\hline Error & 3 & 175 & 58 & & \\
\hline Total & 15 & 4515 & & & \\
\hline S=7.638 & R-Sq=96.12 \% & \multicolumn{2}{c|}{ R-Sq(adj) $=80.62 \%$} \\
\hline Level & N & \multicolumn{3}{|c|}{ Mean } & StDev \\
\hline 7 & 1 & \multicolumn{3}{|c|}{33.00} & $\wedge$ \\
\hline 10 & 1 & 13.00 & $\wedge$ \\
\hline 11 & 1 & 19.00 & $\wedge$ \\
\hline 14 & 1 & 16.00 & $\wedge$ \\
\hline 15 & 2 & 22.50 & 3.536 \\
\hline 17 & 1 & 34.00 & $\wedge$ \\
\hline 20 & 1 & 20.00 & $\wedge$ \\
\hline 22 & 1 & 66.00 & $\wedge$ \\
\hline 25 & 1 & 35.00 & $\wedge$ \\
\hline 33 & 1 & 72.00 & $\wedge$ \\
\hline 37 & 2 & 20.00 & 7.071 \\
\hline 40 & 1 & 40.00 & $\wedge$ \\
\hline 53 & 1 & 22.00 & 10.607 \\
\hline
\end{tabular}

Pooled StDev $=7.638$.

Planta Daninha, Viçosa-MG, v. 34, n. 3, p. 485-495, 2016 


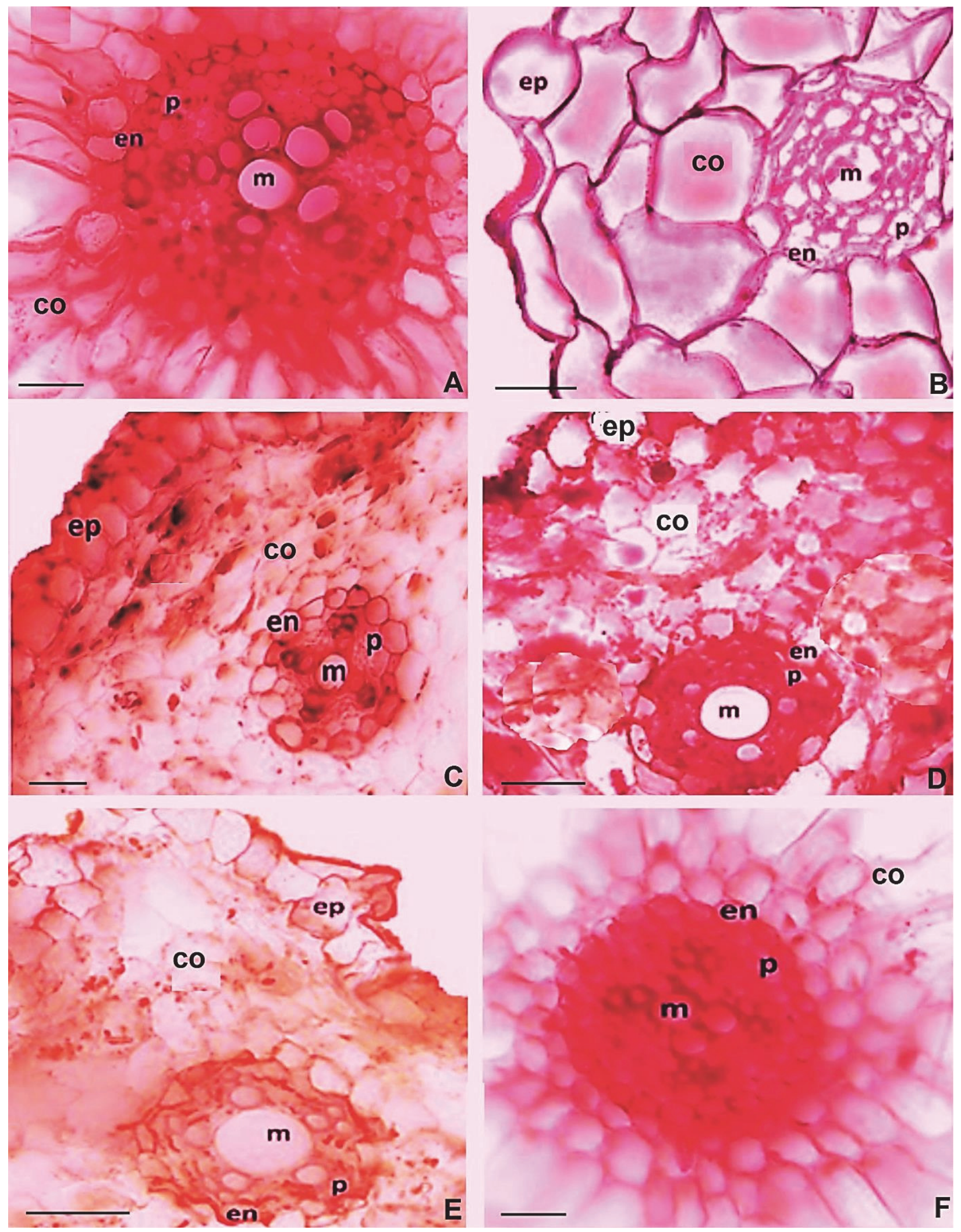

Figure 1 - Root cross sections; A:O. narbonense, B:O. montanum, C:O. wiedemannii, D,E: O.sigmoedeum, F:O. lanceolatum; co: cortex, en: endodermis, ep:epidermis, m:metaxylem, p:pericycle; scale bar $50 \mu \mathrm{m}$. 

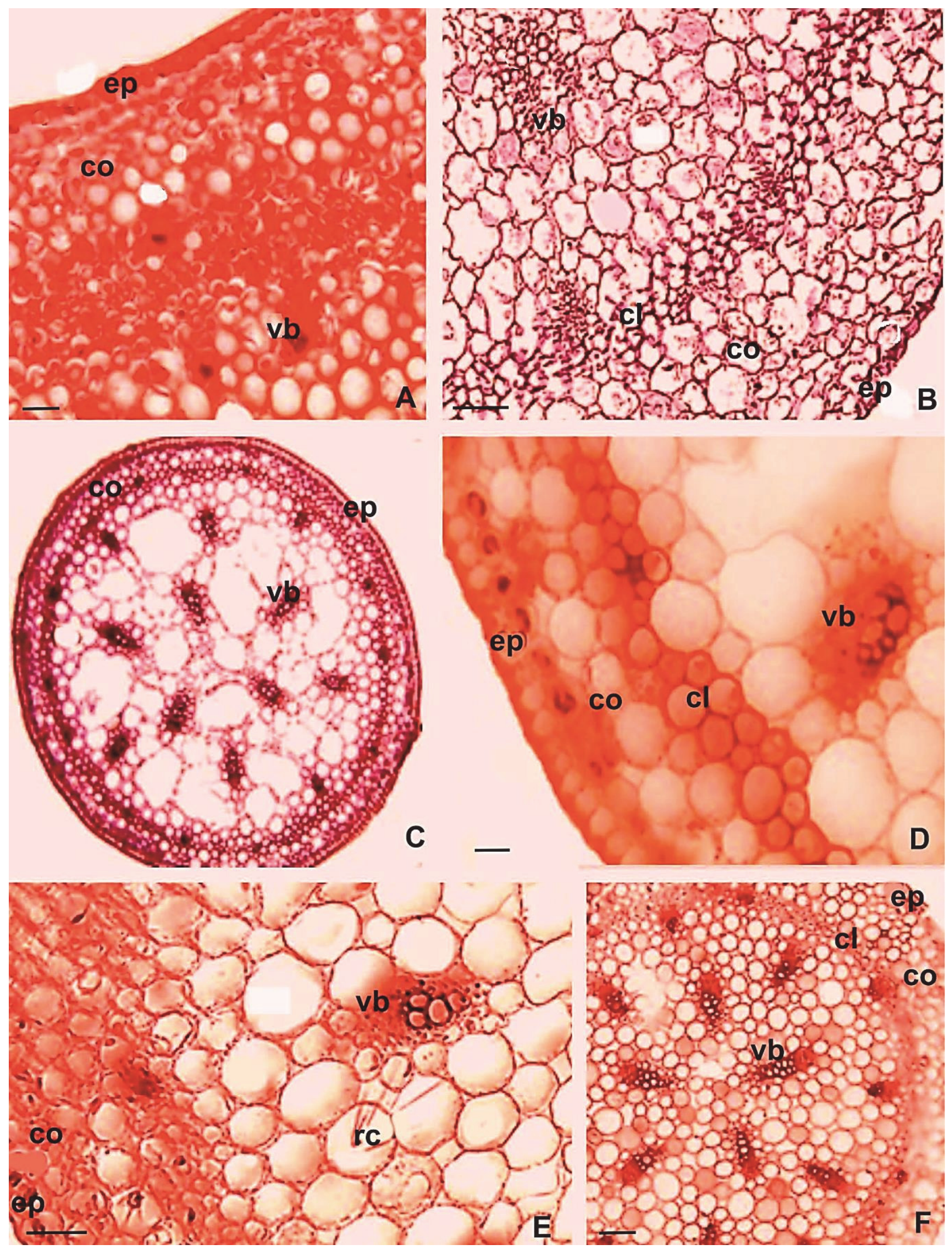

Figure 2 - Stem cross sections; A:O. narbonense, B:O. montanum, C,D:O. wiedemannii, E: O.sigmoedeum, F:O. lanceolatum; co: cortex, cl:collenchyma, ep:epidermis, rc:raphide crystals, vb:vascular bundle; scale bar $50 \mu \mathrm{m}$. 


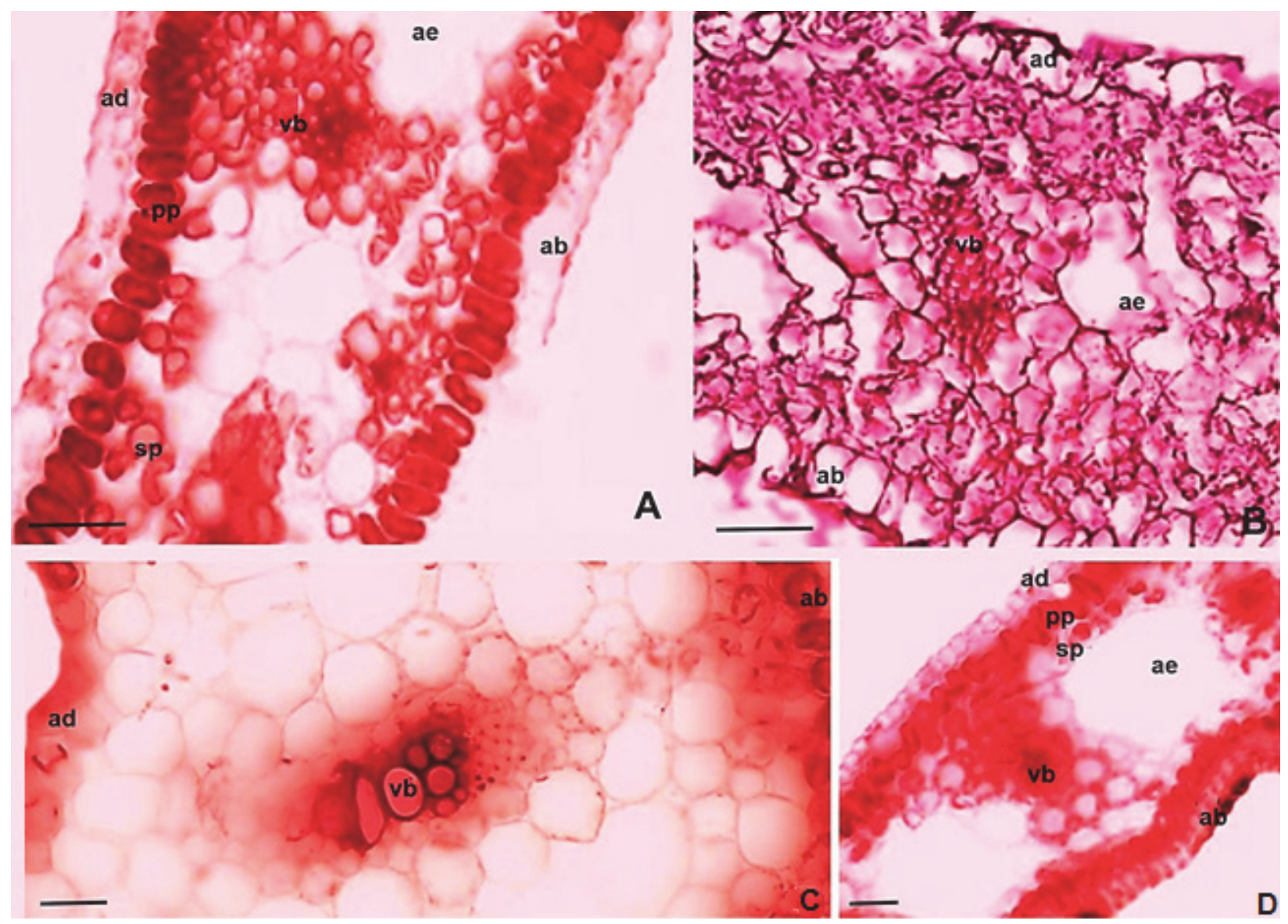

Figure 3 - Leaf cross sections; A:O. narbonense, B:O. montanum, C: O.sigmoedeum, D:O. lanceolatum; ab: abaxial epidermis, ad: adaxial epidermis, ae:aerenchyma, pp:palisade parenchyma, sp:spongy parenchyma vb:vascular bundle; scale bar $50 \mu \mathrm{m}$.

differences. The mesophyll in O. montanum and O.sigmoedeum species is unifacial while it is equifacial in other taxa. The mesophyll in O. narbonense, O. wiedemanniivar wiedemannii, O. lanceolatum. differentiates as palisade and spongy parenchyma. The palisade parenchyma is present in both the adaxial and abaxial side. Also, the mesophyll in O. lanceolatum contains larger aerenchyma between vascular bundles in spongy parenchyma.

\section{REFERENCES}

Coskuncelebi K., Kandemir A., Beyazoglu O. Scanning electron microscopic examination of the seeds of Ornithogalum (Liliaceae) species distributed in Black Sea region of Turkey. Biol Bratislava. 2000:55:397-401.

Coskuncelebi K., Kandemir A., Beyazoglu O. Numerical taxonomic study on Ornithogalum subg. Ornithogalum (Liliaceae) in Black sea region of Turkey. Biol Bratislava. 2002;57:449-54.

Planta Daninha, Viçosa-MG, v. 34, n. 3, p. 485-495, 2016
Cullen J. Ornithogalum L. In: Davis P.H., editor. Flora of Turkey and the East Aegean Islands. Edinburgh: Edinburgh University Press, 1984. v.8. p.227-44

Dalg 1 C G., Dane F., Aksoy O. A new record for the flora of Turkey: Ornithogalum boucheanum (Hyacinthaceae) In: Ivanova D., editor. Plant, fungal and habitat diversity investigation and conservation. Proceedings of 4 th. Balkan Botanical Congress; 2006; Sofia. Sofia, Bulgária: Institute of Botany, 2009. p.169-73.

Dalg1c G., Özhatay N. The genus Ornithogalum (Liliaceae) and its karyotype variation in European Turkey. Bocconea. 1997;5:743-7.

Davis P.H., Mill R.R., Tan K. Ornithogalum L. In: Davis P.H., editor. Flora of Turkey and the East Aegean Islands. Edinburgh: University Edinburgh, 1988. p.225-6. v.10 (Supplement)

Franceschi V.R., Nakata P.A. Calcium oxalate in plants: formation and function. Ann Rev Plant Biol. 2005;56:4171. 
Garbari F. et al. The genus Ornithogalum L. (Hyacinthaceae) in Italy, XIV: towards a redefinition of infrageneric taxa, with new proposals. Bocconea. 2003;16:269-81.

Kamari G., Blanché C., Garbari, F., editors. Mediterranean chromosome number reports - 16. Fl. Medit. 2006;16:385455 .

Lynch A.H., Rudall P.J., Cutler D.F. Leaf anatomy and systematic of Hyacinthaceae. Kew Bull. 2006;61:145-59.

Moret J. Numerical taxonomy applied to a study of some ploidy levels within the Ornithogalum umbellatum complex (Hyacinthaceae) in France. Nordic J Bot. 1992;12:183-95.

Moret J., Favereau Y., Gorenflot R.A. biometric study of the Ornithogalum umbellatum (Hyacinthaceae) complex in France. - Plant Syst. Evol. 1991;175:73-86.

Moret J., Galland N. Phenetic, biogeographical, and evolutionary study of Ornithogalum subg. Heliocharmos (Hyacinthaceae) in the western Mediterranean basin. Plant Syst Evol. 1992;181:179-202.

Øvstedal D.O. A multivariate comparison between Ornithogalum chionophilum Holmboe, O. lanceolatum Labill. and O. montanum Cyr., based on chemometric and morphometric data. Candollea. 1991;46:399-406.

Özhatay N. Ornithogalum L. In: Guner A. et al., editors. Flora of Turkey and the East Aegean Islands. Edinburgh: Edinburgh University, 2000. p.233-37. v.11 (Supplement)
Peruzzi L., Passalacqua N.G. Biosystematic and taxonomic considerations about Italian units of the genus Ornithogalum (Hyacinthaceae) showing reflexed pedicels. Webbia. 2002;57:193-216.

Speta F. Ornithogalum gussonei Ten., O. collinum Guss. und O. exscapum Ten., drei häufig verkannte, aus Italien beschriebene Arten (Hyacinthaceae). Phyton Horn. 1990;30:97-171.

Speta F. Ornithogalum euxinum Speta (a" O. byzantinum Azn., Hyacinthaceae), eine wiederentdeckte Art aus dem Norden der Türkey. Candollea. 1990a;45:447-62.

Tornadore N., Marcucci R., Garbari F. Ornithogalum umbratile (Hyacinthaceae), a new species from Gargano's Promontory, southeastern Italy. Taxon. 2003;52:577-82.

Uysal T., Ertugrul K., Dural H. A new species of Ornithogalum (Liliaceae) from South Anatolia, Turkey. Bot J Linn Soc. 2005;148:501-4.

van Raamsdonk L.W.D., Heringa J. Biosystematic studies on the umbellatum-angustifolium complex in the genus Ornithogalum (Liliaceae). III. Morphological analysis.

Nordic J Bot. 1987;7:631-7.

Varol O. Ornithogalum mekselinae (Liliaceae), a new species from Southwestern Anatolia, Turkey. Nord J Bot. 2008;23:607-9.

Zahariadi C. Caractères morphologiques, anatomiques et biologiques dans la taxonomie du + genre Ornithogalum. Rev Biol. 1962:7:1-41. 